\title{
REFLEXIÓN SOBRE LA \\ IMPORTANCIA DEL FACTOR \\ PSICOLÓGICO EN LA \\ REDUCCIÓN DE DESERCIÓN \\ ESTUDIANTIL
}

\author{
Vanessa Aguirre Flores
}

\begin{abstract}
RESUMEN
La deserción estudiantil es un fenómeno de carácter multifactorial que se da tanto en el nivel escolar como en el nivel superior. Este fenómeno es un problema que debe preocupar a todos aquellos involucrados en el ámbito educativo, así como al Estado por las pérdidas que esta ocasiona. En el presente artículo se mencionan los factores que inciden en la deserción, dando un mayor énfasis al factor psicológico como uno de los principales predictores de este fenómeno. Frente a lo anterior, se hace una reflexión sobre lo relevante que es abordar este factor predictor de la deserción, y así lograr reducir sus índices, proponiendo acciones que permitan a los estudiantes enfrentar con éxito los cambios que implica el inicio de una nueva etapa como es la educación superior.
\end{abstract}

\section{PALABRAS CLAVE}

Deserción estudiantil, factor psicológico, preparación psicológica, educación superior.

\section{ABSTRACT}

Dropout is a multifactorial phenomenon occurring both at the school and at the higher level. This phenomenon is a problem that must concern all those involved in the educational area, as well as the state because of the losses it causes. This article refers the factors that affect desertion, giving greater emphasis to the psychological factor as one of the main predictors of this phenomenon. So, a reflection is made on how relevant it is to address this predictor of desertion, and thus achieve reduction of their indexes, proposing actions that allow students to successfully face the changes involved in the beginning of a newstage as is higher education.

\section{KEYWORDS}

Dropout, psychological factor, psychological preparation, higher education.

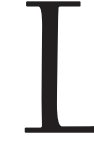

a educación es considerada un aspecto muy importante en el desarrollo de una sociedad; sin embargo, existen problemas que dificultan su objetivo. Una de las preocupaciones en este ámbito y sobre todo a nivel de Latinoamérica, es la deserción estudiantil del nivel superior (UNESCO 2004).

En la actualidad se han realizado diversas investigaciones a nivel internacional y nacional sobre esta problemática, teniendo como resultado modelos de deserción que evidencian variable, las cuales son agrupadas en factores psicológicos, académicos, socioeconómicos e institucionales (Baquero, citado en Rivas, M. 2002).

De los factores mencionados que están asociados a la deserción, se considera para este artículo, al factor psicológico de gran importancia, pues de las investigaciones revisadas, estas coinciden en su mayoría, siendo uno de los factores que mayor incidencia tiene, seguido por otros como el socioeconómico.

Aun cuando los resultados de las diferentes investigaciones muestren los factores de deserción, existen pocas publicaciones sobre el abordaje de su tratamiento, lo que podría indicar que aún sigue siendo un gran desafío su reducción. Si bien es cierto, el abordaje que requiere compete en primera instancia a las instituciones educativas involucradas, también se asume que es necesaria la participación del Estado no solo cuando el problema ya se evidencie sino también desde una etapa anterior; es decir, intervenir desde los últimos años del nivel secundario. 
En nuestro país existen estudios que ponen de manifiesto la situación de la educación respecto a esta variable, así pues, Logros (2011) concluye que la deserción universitaria en el Perú alcanza una tasa del 17\% (50 mil jóvenes) y que los factores comunes, incluyendo no sólo el nivel superior universitario sino también el no universitario, muestran que hasta un 30\% de alumnos procede a abandonar su centro de estudios antes de terminar el primer semestre, según la Consultora en Marketing Flanqueo (2013).

Esta situación implica que demos una mirada de análisis y reflexión sobre cómo abordar tal problema. En tanto, es importante conocer cómo definen a la deserción algunos autores, teniendo en conocimiento que es un tema que se viene trabajando desde décadas atrás hasta la actualidad.

Tinto (1982), considera que un desertor es aquel individuo que siendo estudiante de una institución de educación superior no presenta actividad académica durante tres semestres académicos consecutivos. Mientras, Goicovic (2002) define a la deserción como el abandono que realiza el alumno de todas sus labores escolares con el objetivo de no regresar. Por su parte, una definición más actual es la de Himmel (2005), quien refiere que la deserción es el abandono prematuro que realiza el estudiante frente a un programa de estudios antes de alcanzar el título o grado y considera un tiempo suficientemente largo para descartar posibilidades de que el estudiante retorne y se reincorpore.

En cuanto a los factores que influyen en el origen de este problema, existen modelos, los cuales ayudan a tener una mayor claridad de las diversas causas que influyen en la toma de decisión de desertar en el estudiante. Se puede mencionar a Baquero (citado en Rivas, M. 2002), como aquel que según las diferentes causas logra clasificarlas en los siguientes factores como: los psicológicos (motivaciones, desadaptación, expectativas, además de otros como edad, salud, etc.), académicos (rendimiento, estrategias y hábitos de estudio, etc.), socioeconómicos e institucionales (calidad de docentes, eficiencia administrativa, etc.).
Estos modelos explicativos son, a su vez, respaldados por investigaciones como la de Sánchez (2012), quien menciona que los factores que influyen en la deserción son en primer lugar los de índole personal, seguido por el económico, académico y social. Asimismo, Abunzer (2009) recoge como resultados que el factor personal y económico están relacionados con la deserción; por otro lado, Quispe y Álvarez (2009) señalan en su investigación que existen variables que corresponde al ámbito personal /psicológico, las cuales han influido sobre el abandono académico.

Además de los anteriores, se citará un aporte más que es el de Medrano et al. (2000) donde respalda al factor psicológico (creencias irracionales) como influyente sobre la deserción estudiantil.

Habiendo hecho una revisión breve de la literatura acerca de la deserción y sus factores, se puede asumir una constante en las investigaciones mencionadas, que son las causas de orden psicológico las que están influyendo en la aparición de este problema en cuestión. Si bien es cierto el factor psicológico no actúa de forma aislada es válido inferir que si se hace un abordaje completo podría reducir el índice de deserción.

Ante esta evidencia, también surge el plantearse algunas interrogantes que ayudarán a sensibilizar sobre esta situación. La primera pregunta es ilas instituciones de educación superior no están listas para recibir a los estudiantes brindándoles un adecuado proceso en su integracióny adaptación? ¿Será acaso que en los centros de EBR (Educación Básica Regular) no se contempla como aspecto importante la formación de competencias personales básicas para afrontar cambios a una vida universitaria? o finalmente iserá responsabilidad del Estado monitorear el proceso de admisión de estas instituciones?

Respecto a la primera pregunta, tan sólo tendríamos que dar una mirada atrás y comparar que hace cinco décadas existían 10 universidades; sin embargo, en la actualidad según la Asamblea Nacional de Rectores, se tiene aproximadamente 129 universidades de diversos tipos, las cuales tienen que ser reguladas ya que varias de ellas aún no son autorizadas en su totalidad para funcionar como tales, lo que podría indicar que el objetivo 
real y principal para el cual fueron creadas es de orden lucrativo. Este incremento de universidades trae consigo también el deseo de mayor captación de e estudiantes, siendo en la actualidad eliminado los famosos exámenes de admisión pues sería un obstáculo para incrementar sus ingresos económicos, lo cual no sería tan grave si es que así como se preocupan por la publicidad y tener mayor cantidad de estudiantes, lograran hacerlo de forma adecuada considerando aspectos importantes como la guía y orientación en la elección adecuada de profesión elegida, desarrollando talleres que fortalezcan sus competencias personales, acompañarlos en el proceso de integración y adaptación.

En cuanto a la segunda interrogante, el papel que cumplen los colegios respecto a la preparación que brindan a los estudiantes de los últimos grados, en su mayoría se enfocan sólo en el aspecto del rendimiento académico (simulacros de exámenes de admisión, más horas de estudio en los diferentes cursos que están ligados a la carrera que estudiarán), dejando de lado un factor importante como es psicológico, el cual debería fomentarse en paralelo para desarrollar fortalezas que le faciliten su integración y adaptación.

Finalmente, en relación a la última pregunta, se puede decir que aun cuando en estos tres últimos años se viene trabajando más en el aspecto de regular la calidad de enseñanza en las universidades con la nueva Ley Universitaria 30220 , se considera que todavía no logra establecerse medidas que regulen el proceso de admisión de los estudiantes en las diferentes instituciones educativas del nivel superior.

En base a lo mencionado anteriormente, se puede sugerir posibles acciones de intervención para la reducción de la deserción abordándolo desde el factor psicológico. Así pues, en lo que respecta a las instituciones educativas del nivel superior y de EBR, un abordaje adecuado disminuiría los índices de deserción. Este trabajo estaría enfocado, en primer lugar, en brindarle una adecuada información y orientación a los postulantes o alumnos que deseen inscribirse a una determinada carrera profesional, así pues podrían bien desarrollar ferias, charlas o talleres de orientación vocacional para reafirmar al estudiante sobre su elección o en todo caso aclarar dudas.

Por otro lado, la siguiente acción a ejecutar sería en la etapa de estudio de los primeros ciclos de la profesión, con el que se debería contemplar la presencia de personal capacitado para asumir la función de tutor quien acompañe al estudiante en este proceso de integración, desarrollando sesiones para el logro de competencias personales, además de ello poder fomentar el sentimiento de pertenencia a la institución. Por otro lado, también resultaría beneficioso, elaborar programas de retención como actualmente vienen realizando algunas instituciones, las cuales se centran en hacer seguimiento al estudiante brindándole apoyo psicológico y también facilidades de pago (que si bien es cierto este último es un factor distinto al abordado en esta sección, también se tiene que recordar que no actúan de forma independiente ni aislada) que recaen en fomentar en el estudiante este sentimiento de pertenencia al percibir que la institución muestra interés y preocupación por su bienestar.

La intervención del Estado estaría en función a desarrollar actividades reguladoras de los procesos de admisión, así como en el apoyo hacia las instituciones educativas para el desarrollo de temas blandos, así como el respaldo en la elaboración de programas de retención.

Para finalizar con este artículo, se puede mencionar las siguientes conclusiones:

A pesar de los diferentes factores que inciden en el problema de la deserción, el factor psicológico es uno de los más frecuentes que amerita por lo tanto una inmediataintervención.

El incremento de instituciones de educación superior, fomenta entre ellas un deseo de competir en busca de clientes, a través de diversas acciones de publicidad y marketing; olvidándose en muchos casos que la tasa de deserción es un indicador en cuanto a la calidad institucional, y entender que es primordial enfocar todos los medios necesarios para atender y ayudar al 
estudiante con dificultades.

Las instituciones de educación superior cumplen un rol importante en el proceso adecuado de adaptación del estudiante a esta nueva etapa académica para lograr la reducción del fenómeno de la deserción.

\section{REFERENCIAS}

Rivas, S. (2002). Rendimiento académico y deserción estudiantil en pregrado: Caso Universidad Católica Andrés Bello (Tesis de Maestría). Recuperado de http://biblioteca2 .ucab.edu.ve/anexos/biblioteca/marc/texto/A AP6463.pdf

Logros (2011). Portal del Postulante. www.logros.edu.pe

Tinto, Vincent. La deserción en la educación superior: Síntesis de las bases teóricas de las investigaciones recientes. Review of Educational Research, Vol. 45, № 1, USA, 1975, pp. 89-195 (trad. de Carlos María de Allende).

Abensur S. (2009). Factores socioeconómicos y personales relacionados con la deserción estudiantil en la Escuela de Negocios Internacionales de la Facultad de Ciencias
La deserción estudiantil como consecuencia de aspectos psicológicos es un problema que debe ser abordado de forma preventiva desde los colegios, así como desde el interior mismo de la institución de educación superior.
Económicas y Negocios de la Universidad Nacional de la Amazonía Peruana (Tesis de Maestría). Recuperado de http:// www.unsa.edu.pe/ofrrpp/informe/radiografi a-de-la-universidad-peruana-2/

Goicovic Donoso, I. (2002). Educación, deserción escolar e integración laboral juvenil. Última década. Recuperado de http://www.redalyc. org/articulo.oa?id=19501602

Quispe y Alvarez (2009). Factores socioeconómicosacadémicos que influyen en la deserción estudiantil en Institutos Superiores Tecnológicos de Moquegua.

Medrano, Galleano, Galera y Valle (2010). Creencias irracionales, rendimiento y deserción académica en ingresantes universitarios. Recuperado de: http://www.redalyc.org /html/686/68617161007/ 